\title{
Quantum spherical spin glass: Supersymmetry and annealing
}

\author{
Pedro Castro Menezes and Alba Theumann \\ Instituto de Física, Universidade Federal do Rio Grande do Sul, Avenue Bento Gonçalves 9500, 91501-970 Porto Alegre, RS, Brazil
}

(Received 3 November 2005; revised manuscript received 16 August 2006; published 31 January 2007)

\begin{abstract}
We show that the effective action of the quantum spherical spin glass is invariant under a generalized form of Becchi-Rouet-Stora-Tyutin supersymmetry. The Ward identities associated with this invariance indicate that the spin glass order parameter must vanish, and as a result the annealed average is exact in this model. We present results for the free energy, entropy, and specific heat. Due to quantum effects, the entropy remains finite and the specific heat vanishes at zero temperature. Results for the phase diagram coincide with those obtained by different formalisms. At zero temperature we derive the scaling behavior with frequency of the dynamical susceptibility.
\end{abstract}

DOI: 10.1103/PhysRevB.75.024433

PACS number(s): 75.10.Nr, 75.10.Jm, 64.70.Pf

\section{INTRODUCTION}

In a previous publication ${ }^{1}$ the classical spherical spin glass was analyzed by using supersymmetry methods ${ }^{2,3}$ and it was pointed out that the model was "anomalous" because the order parameter $q$ that measures the replica's overlap was vanishing and the configurational average of any number of replicas gave the correct known result; ${ }^{4}$ then, the annealed approximation with only one replica was also correct. More recent work on the calculation of the complexity, or logarithm of the density of states of a certain energy, of the $p$-spherical spin glass, $p \geqslant 3,{ }^{5,6}$ and of the SherringtonKirkpatrick model ${ }^{7}$ shows that the annealed average gives the correct answer when the action is invariant under the generalized Becchi-Rouet-Stora-Tyutin (BRST) supersymmetry. ${ }^{8}$

In the present paper we first demonstrate how the generalized BRST supersymmetry transformation applies to the functional integral formulation of the quantum spherical spin glass. We also discuss how this internal invariance leads to Ward identities that result in the vanishing of the replica overlap $q$. This result had been obtained previously for the classical spin glass ${ }^{1}$ and justifies the performance of the annealed configurational average $\mathrm{e}^{5-7}$ also in the quantum spherical spin glass. The model was proposed earlier in Ref. 9 and was solved by using the distribution of eigenvalues of a Gaussian random matrix. Later, the interest in this model was renewed because it corresponds to the infinite- $M$ limit of the spin glass of $M$-component quantum rotors and it was studied ${ }^{10}$ by using the replica method in a replica-symmetric theory. Both theories give the same phase diagram, although thermodynamic quantities such as free energy and specific heat are not discussed. On the other hand, the spin-glass model of quantum rotors is equivalent to the Ising spin-glass model in a transverse field. ${ }^{11} \mathrm{~A}$ formulation for quantum spherical spins with a different temporal dependence was presented for multispin interactions. ${ }^{12}$ Critical properties were analyzed in Ref. 13, and applications to physical systems were presented in Refs. 14 and 15. All these papers coincide in that the quantum spherical model presents a finite entropy and vanishing specific heat at low temperature, while the classical model presents a negative infinite entropy and constant specific heat.
We study the quantum spherical spin glass in the annealed average by using a functional integral formulation derived by using Feynman's prescription. ${ }^{16,17}$ In Sec. II we present the general formalism and discuss the supersymmetry transformation that leaves invariant the action together with the corresponding Ward identities, ${ }^{18}$ while in Sec. III we discuss the results. We are careful to normalize the free energy so as to recover a finite classical limit when the rotors moment of inertia $I \rightarrow \infty$. In this way the phase diagram, which is calculated from the spherical condition, coincides with previous studies. ${ }^{9,10}$ In the classical limit $I \rightarrow \infty$ the entropy diverges at $T=0$ in the typical fashion of the classical spherical model, ${ }^{1,4}$ with the important difference that it remains finite in the quantum regime for finite values of the rotors moment of inertia. The specific heat shows the usual discontinuity at the critical temperature but it vanishes at $T=0$ in the quantum regime. We derive scaling laws for the dynamical susceptibility, in agreement with previous results. ${ }^{10} \mathrm{We}$ present in the Appendix some useful relations among Grassmann variables without proof, while we refer the reader to the original work ${ }^{2}$ for rigorous results.

\section{SUPERSYMMETRY AND ANNEALING}

We consider a spin glass of quantum rotors ${ }^{10}$ with moment of inertia $I$ in the spherical limit with Hamiltonian

$$
\mathcal{H}_{S G}+\mu \sum_{i} S_{i}^{2}=\frac{1}{2 I} \sum_{i} P_{i}^{2}-\frac{1}{2} \sum_{i, j} J_{i j} S_{i} S_{j}+\mu \sum_{i} S_{i}^{2},
$$

where the spin variables at each site are continuous $-\infty$ $<S_{i}<\infty$ and we introduced the canonical momentum $P_{i}$ with commutation rules

$$
\left[S_{j}, P_{k}\right]=i \delta_{j, k} .
$$

The sum in Eq. (1) runs over sites $i, j=1, \ldots, N$. The coupling $J_{i j}$ in Eq. (1) is an independent random variable with the Gaussian distribution

$$
P\left(J_{i j}\right)=e^{-J_{i j}^{2} N / J^{2}} \sqrt{\frac{N}{\pi J^{2}}},
$$

while the chemical potential $\mu$ is a Lagrange multiplier that ensures the mean spherical condition 


$$
-\frac{\partial\langle\ln \mathcal{Z}\rangle}{\partial(\mu)}=\sum_{i} \int_{0}^{\beta} d \tau\left\langle S_{i}^{2}\right\rangle=\beta N
$$

and $\beta=1 / T$ is the inverse temperature. We work in units where the Boltzmann constant $k_{B}=\hbar=1$ and $\mathcal{Z}$ is the quantum partition function

$$
\mathcal{Z}=\operatorname{Tr} \exp \left[-\beta\left(\mathcal{H}_{S G}+\mu \sum_{i} S_{i}^{2}\right)\right]
$$

The partition function at zero field can be expressed as a functional integral ${ }^{16,17}$

$$
\mathcal{Z}=\int \prod_{i} \mathcal{D} S_{i} \exp \left(-\mathcal{A}_{\mathcal{O}}-\mathcal{A}_{\mathcal{S G}}\right)
$$

where the noninteracting action $\mathcal{A}_{\mathcal{O}}$ is given by

$$
\mathcal{A}_{\mathcal{O}}=\int_{0}^{\beta} d \tau \sum_{i}\left[\frac{I}{2}\left(\frac{\partial S_{i}}{\partial \tau}\right)^{2}+\mu S_{i}^{2}(\tau)-H_{i}(\tau) S_{i}(\tau)\right]
$$

and the interacting part

$$
\mathcal{A}_{\mathcal{S G}}=\frac{1}{2} \sum_{i, j} J_{i j} \int_{0}^{\beta} d \tau S_{i}(\tau) S_{f}(\tau) .
$$

After performing a Fourier transformation in time and taking into account the reality of the fields $S_{i}(\tau)$ the generating functional can be written in terms of the real components $R_{i}(n)$ of the Fourier transforms $S_{i}\left(\omega_{n}\right)=S_{i}^{*}\left(-\omega_{n}\right)$, where $\omega_{n}$ $=\frac{2 \pi n}{\beta}$ are boson Matsubara's frequencies. As was shown in Ref. 1 the correlation functions can be obtained from the generating functional for two replicas:

$$
\begin{gathered}
\mathcal{W}(H)=\prod_{n \geqslant 0}\left\|\Gamma_{i j}(n)\right\| \int \prod_{i \alpha n \geqslant 0} d R_{i \alpha}(n) \\
\exp \left(-\sum_{\alpha n \geqslant 0} \sum_{i j} \Gamma_{i j}(n) R_{i \alpha}(n) R_{j \alpha}(n)+\sum_{i \alpha} H_{i \alpha}(n) R_{i \alpha}(n)\right),
\end{gathered}
$$

where the index $\alpha=1,2$, the $H_{i \alpha}(n)$ are auxiliary fields, and

$$
\Gamma_{i j}(n)=\left(I \beta \omega_{n}^{2}+2 \beta \mu\right) \delta_{i j}-J_{i j} \beta .
$$

By using the results in the Appendix, the determinant $\left\|\Gamma_{i j}(n)\right\|$ may be expressed with the help of the auxiliary Grassmann fields $\chi^{*}$ and $\chi$ and $\mathcal{W}$ can be written

$$
\begin{aligned}
\mathcal{W}\left(H_{\alpha}, \gamma\right)= & \prod_{n \geqslant 0} \int \prod_{i n \geqslant 0} d \chi_{i}^{*}(n) d \chi_{i}(n) \prod_{\alpha} d R_{i \alpha}(n) \exp -\left\{\sum _ { n \geqslant 0 } \left[\sum_{i j} \Gamma_{i j}(n)\left(\frac{\chi_{i}^{*}(n) \chi_{j}(n)+\chi_{j}^{*}(n) \chi_{i}(n)}{2}+\sum_{\alpha} R_{i \alpha}(n) R_{j \alpha}(n)\right)\right.\right. \\
& \left.\left.+\sum_{i}\left(\frac{\gamma_{i}^{*}(n) \chi_{i}(n)+\chi_{i}^{*}(n) \gamma_{i}(n)}{2}+\sum_{\alpha} H_{i \alpha}(n) R_{i \alpha}(n)\right)\right]\right\},
\end{aligned}
$$

where $\chi_{i}^{*}(n)$ and $\chi_{i}(n)$ are complex anticommuting Grassmann variables while the $R_{i, \alpha}(n), \alpha=1,2$, are real commuting variables and we introduced two extra Grassmann auxiliary fields $\gamma_{i}$ and $\gamma_{i}^{*}$. As the $\Gamma_{i j}$ are symmetric, when the auxiliary fields are set equal to zero the functional is invariant under the supersymmetry transformation

$$
\begin{gathered}
\chi_{i}^{\prime *}(n) / \sqrt{2}=\chi_{i}^{*}(n) / \sqrt{2}+\epsilon^{*} \sum_{\alpha} R_{i, \alpha}(n) / \sqrt{2}, \\
\chi_{i}^{\prime}(n) / \sqrt{2}=\chi_{i}(n) / \sqrt{2}+\epsilon \sum_{\alpha} R_{i, \alpha}(n) / \sqrt{2}, \\
R_{i, \alpha}(n)^{\prime}=R_{i, \alpha}(n)-\frac{\epsilon^{*} \chi_{i}(n)}{2}+\frac{\epsilon \chi_{i}^{*}(n)}{2},
\end{gathered}
$$

where $\epsilon$ is a complex Grassmann variable and we adopt the convention for complex conjugation, ${ }^{2} \epsilon^{* *}=-\epsilon,\left(\epsilon \chi_{i}^{*}\right)^{*}$ $=\epsilon^{*} \chi_{i}^{* *}=-\epsilon^{*} \chi_{i}$; then, $R_{i, \alpha}(n)^{\prime}$ is effectively a real variable. The transformation differs infinitesimally from unity, and $\epsilon^{*} \epsilon$ can be neglected, although this concept is somehow meaningless in the case of Grassmann variables. Now it is conve- nient to switch to the superalgebra notation ${ }^{2}$ of the Appendix and to introduce the supervectors for the auxiliary fields,

$$
\begin{gathered}
\underline{J}_{i}=\left(\begin{array}{c}
H_{i 1} \\
H_{i 2} \\
\gamma_{i} / \sqrt{2} \\
\gamma_{i}^{*} / \sqrt{2}
\end{array}\right), \\
\underline{S J}_{i}^{\dagger}=\left(H_{i 1} H_{i 2} \gamma_{i}^{*} / \sqrt{2}-\gamma_{i} / \sqrt{2}\right),
\end{gathered}
$$

and for the field variables,

$$
\begin{gathered}
\underline{\varphi}_{i}=\left(\begin{array}{c}
R_{i 1} \\
R_{i 2} \\
\chi_{i} / \sqrt{2} \\
\chi_{i}^{*} / \sqrt{2}
\end{array}\right), \\
\underline{S \varphi_{i}^{\dagger}}=\left(R_{i 1} R_{i 2} \chi_{i}^{*} / \sqrt{2}-\chi_{i} / \sqrt{2}\right) .
\end{gathered}
$$

In this way the functional $\mathcal{W}$ in Eq. (11) may be written in compact form 


$$
\begin{gathered}
\mathcal{W}(J)=\prod_{n \geqslant 0} \int \prod_{i n \geqslant 0} d \chi_{i}^{*}(n) d \chi_{i}(n) \prod_{\alpha} d R_{i \alpha}(n) \\
\exp \left[-1 / 2 \sum_{i j} \underline{S \varphi_{i}^{\dagger}} \mathbf{M}_{i j} \underline{\varphi}_{i}+\sum_{i} \underline{S J}_{i}^{\dagger} \underline{\varphi}_{i}\right],
\end{gathered}
$$

which may be formally integrated to give

$$
\mathcal{W}(J)=\exp \left[1 / 2 \sum_{i j n} \underline{S J_{i}^{\dagger}} \mathbf{Q}_{i j} J_{j}\right],
$$

with the $4 \times 4$ supermatrices

$$
\mathbf{Q}_{\mathrm{ij}}=\left(\begin{array}{cccc}
q_{11} & q_{12} & \theta_{13} & \theta_{14} \\
q_{21} & q_{22} & \theta_{23} & \theta_{24} \\
\theta_{31} & \theta_{32} & v_{33} & 0 \\
\theta_{41} & \theta_{42} & 0 & v_{44}
\end{array}\right)_{\mathbf{i j}}
$$

By symmetry we should have

$$
\begin{gathered}
q_{11}=q_{22}=\left\langle R_{i \alpha} R_{i \alpha}\right\rangle=v_{33}=v_{44}=\left\langle\chi^{*} \chi\right\rangle, \\
v_{34}=v_{43}=\langle\chi \chi\rangle=\left\langle\chi^{*} \chi^{*}\right\rangle=0 .
\end{gathered}
$$

To derive Ward identities ${ }^{18}$ we consider that, as the system is invariant when the fields are subject to the supersymmetry transformation in Eq. (12),

$$
\underline{\varphi}_{i}^{\prime}=\mathbf{A} \underline{\varphi}_{i},
$$

where

$$
\mathbf{A}=\left(\begin{array}{cccc}
1 & 0 & -\epsilon^{*} / \sqrt{2} & \epsilon / \sqrt{2} \\
0 & 1 & -\epsilon^{*} / \sqrt{2} & \epsilon / \sqrt{2} \\
\epsilon / \sqrt{2} & \epsilon / \sqrt{2} & 1 & 0 \\
\epsilon^{*} / \sqrt{2} & \epsilon^{*} / \sqrt{2} & 0 & 1
\end{array}\right)=\mathbf{1}+\mathbf{D},
$$

then it should be also invariant when we transform the external fields ${ }^{18}$ in Eq. (16):

$$
\begin{gathered}
\underline{J}^{\prime}=\mathbf{A} \underline{J}, \\
{\underline{S J^{\dagger \prime}}}^{=} \underline{S J^{\dagger}} \mathbf{S A}^{\dagger},
\end{gathered}
$$

where the superadjoint $\mathbf{S A}^{\dagger}$ is defined in the Appendix. This condition leads to

$$
\mathbf{S D}^{\dagger} \mathbf{Q}+\mathbf{Q D}=\mathbf{0},
$$

from where we deduce

$$
\begin{aligned}
& v_{33}=q_{11}+q_{12}, \\
& v_{44}=q_{22}+q_{21},
\end{aligned}
$$

and from Eq. (18) we obtain for the overlap between two replicas

$$
q_{12}=q_{21}=0 .
$$

The spin-glass order parameter $q=q_{12}$ and it vanishes identically from Eq. (19), thus making the annealed average exact in the quantum spherical spin glass as was discussed in Ref. 1 for the classical spherical spin glass.

\section{RESULTS}

When performing the annealed average we take the configurational average of $\mathcal{Z}$ in Eq. (6) over the random variables $J_{i j}$ and after splitting the quadratic term with a Gaussian integration we obtain the result

$$
\begin{aligned}
\langle\mathcal{Z}\rangle_{c a}= & \int \mathcal{D} Q\left(\tau-\tau^{\prime}\right) \\
& \times \exp \left[-N\left(\frac{J^{2}}{4} \int_{0}^{\beta} \int_{0}^{\beta} d \tau d \tau^{\prime} Q\left(\tau-\tau^{\prime}\right)^{2}-\Lambda\right)\right],
\end{aligned}
$$

where we assumed time translational invariance and

$$
\begin{aligned}
e^{\Lambda}= & \int \mathcal{D} S(\tau) \exp \left[-\mathcal{A}_{\mathcal{O}}+\frac{J^{2}}{2} \int_{0}^{\beta} \int_{0}^{\beta} d \tau d \tau^{\prime}\right. \\
& \left.\times Q\left(\tau-\tau^{\prime}\right) S(\tau) S\left(\tau^{\prime}\right)\right] .
\end{aligned}
$$

A steepest-descent calculation in Eq. (24) gives

$$
Q\left(\tau-\tau^{\prime}\right)=\frac{1}{N} \sum_{i}\left\langle S_{i}(\tau) S_{i}\left(\tau^{\prime}\right)\right\rangle .
$$

The partition function in Eq. (24) is solved by a time Fourier transformation with the result

$$
\begin{aligned}
\langle\mathcal{Z}\rangle_{c a}= & \mathcal{N}^{N} \prod_{n=0}^{\infty} \int d Q\left(\omega_{n}\right) \exp \left\{-N\left[\frac{(\beta J)^{2}}{2} Q\left(\omega_{n}\right)^{2}\right.\right. \\
& -\frac{\beta H^{*}\left(\omega_{n}\right) H\left(\omega_{n}\right)}{\beta I \omega_{n}^{2}+2 \beta \mu-2(\beta J)^{2} R\left(\omega_{n}\right)} \\
& \left.\left.+\ln \left[\beta I \omega_{n}^{2}+2 \beta \mu-(\beta J)^{2} Q\left(\omega_{n}\right)\right]\right]\right\},
\end{aligned}
$$

where $\omega_{n}=\frac{2 \pi n}{\beta}$ is the boson Matsubara frequency and $Q\left(\omega_{n}\right)$ is the real part of the Fourier transform of $Q(\tau)$. A steepest-

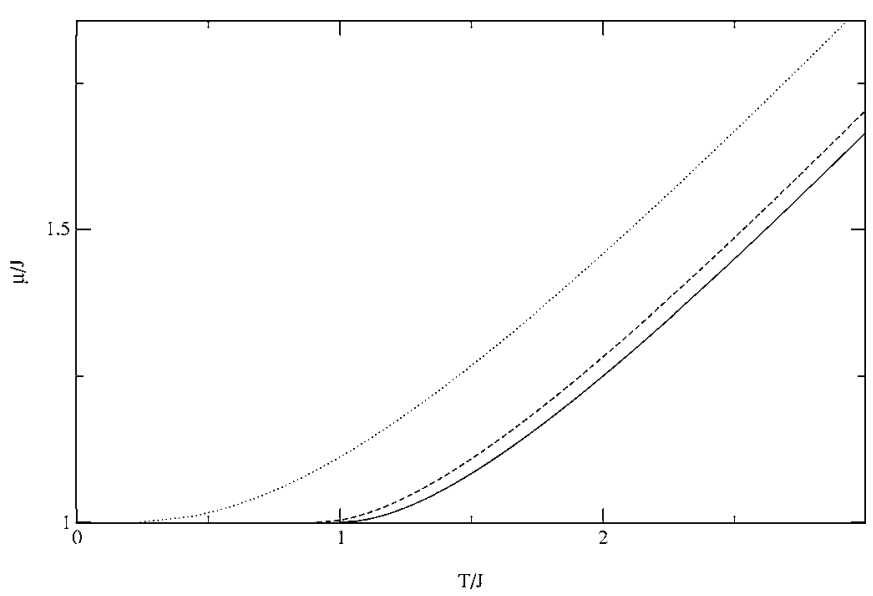

FIG. 1. Chemical potential $\mu$ as a function of temperature for different values of the moment of inertia $I=0.19 \mathrm{~J}$ (dash-dotted line), $I=J$ (dashed line), $I=\infty$ (solid line). 


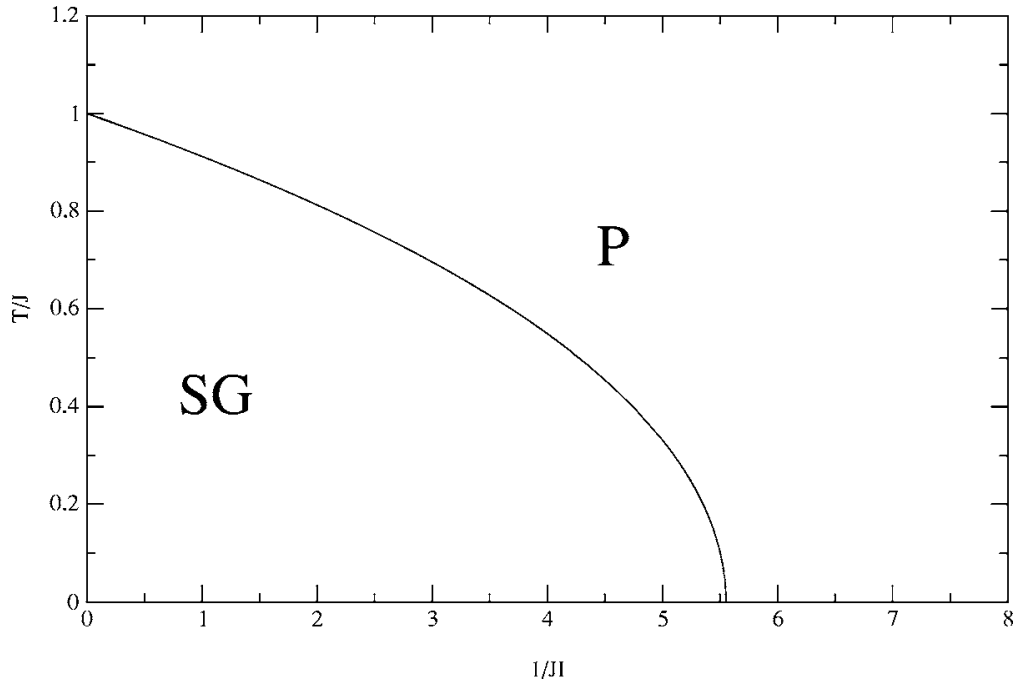

FIG. 2. Phase diagram and critical line $T_{c}(1 / I)$ separating the paramagnetic from the spin glass phase in the $T$ vs $1 / I$ plane. descent evaluation of the integral in Eq. (27) gives, for $H\left(\omega_{n}\right)=0$,

$$
Q\left(\omega_{n}\right)=\left[\beta I \omega_{n}^{2}+2 \beta \mu-(\beta J)^{2} Q\left(\omega_{n}\right)\right]^{-1},
$$

with the solution

$$
Q\left(\omega_{n}\right)=\frac{\beta I \omega_{n}^{2}+2 \beta \mu-\sqrt{\left(\beta I \omega_{n}^{2}+2 \beta \mu\right)^{2}-4(\beta J)^{2}}}{2(\beta J)^{2}},
$$

while the mean spherical condition in Eq. (4) becomes

$$
\sum_{-\infty}^{\infty} Q\left(\omega_{n}\right)=1
$$

Introducing Eq. (29) into Eq. (27) we obtain for the free energy per site at the saddle point

$$
\beta F=\sum_{-\infty}^{\infty}\left(\frac{1}{4}(\beta J)^{2} Q\left(\omega_{n}\right)^{2}-\frac{1}{2} \ln \left[Q\left(\omega_{n}\right)\right]\right)-\beta \mu-\ln (\mathcal{N}) .
$$

The normalization constant $\mathcal{N}=\frac{1}{\sqrt{I}}$ is determined so that we recover a finite limit for the free energy when $I \rightarrow \infty$. Follow- ing standard procedures ${ }^{16}$ we convert sums over frequencies into integrals with the result for the mean spherical condition

$$
\int_{L_{-}}^{L_{+}} d y \sqrt{\left(L_{+}^{2}-y^{2}\right)\left(y^{2}-L_{-}^{2}\right)} \operatorname{coth}\left(\frac{\beta y}{2 \sqrt{I}}\right)=2 \pi J^{2} \sqrt{I},
$$

where

$$
L_{ \pm}^{2}=2 \mu \pm 2 J
$$

and we obtain for the free energy

$$
\begin{aligned}
\beta F= & \frac{1}{\pi J^{2}} \int_{L_{-}}^{L_{+}} d y y \ln \sinh \left(\frac{\beta y}{2 \sqrt{I}}\right) \sqrt{4 J^{2}-\left(2 \mu-y^{2}\right)^{2}}-\beta \mu \\
& -\ln \mathcal{N} .
\end{aligned}
$$

In the classical limit $I \rightarrow \infty$ the integrals in Eqs. (32) and (34) can be performed exactly in terms of hypergeometric functions with the result

$$
\frac{2 \mu_{\text {class }}}{J}=\beta J+\frac{1}{\beta J},
$$

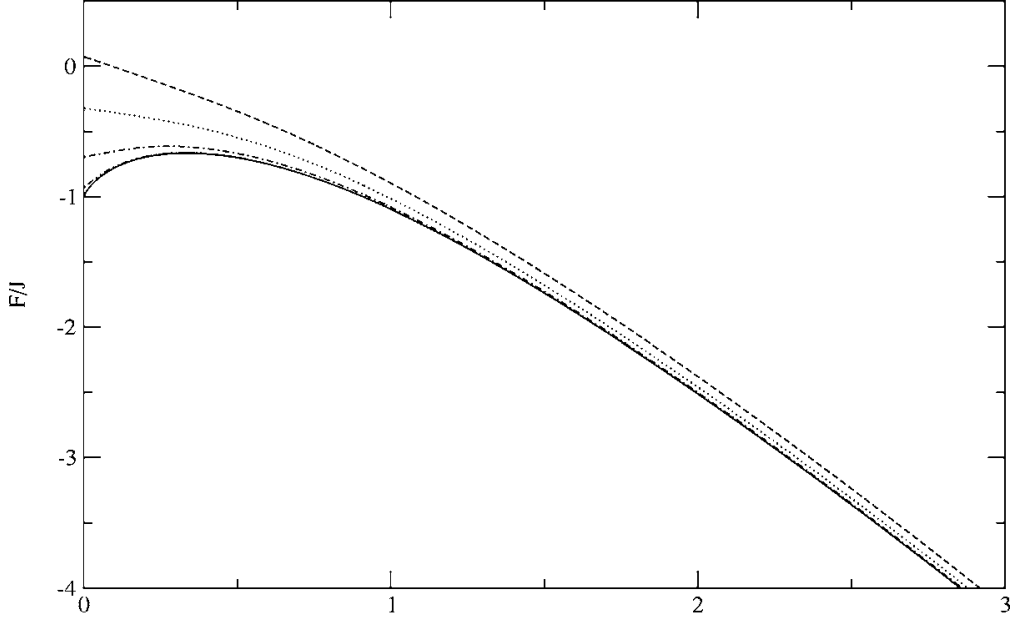

$\mathrm{T} / \mathrm{J}$
FIG. 3. Free energy as a function of temperature for different values of the moment of inertia $I=0.4 J$ (dashed line), $I=J$ (dash-double-dotted line), $I=5 J$ (dash-dotted line), $I=10 J$ (dashdouble-dotted line), $I=\infty$ (solid line). 


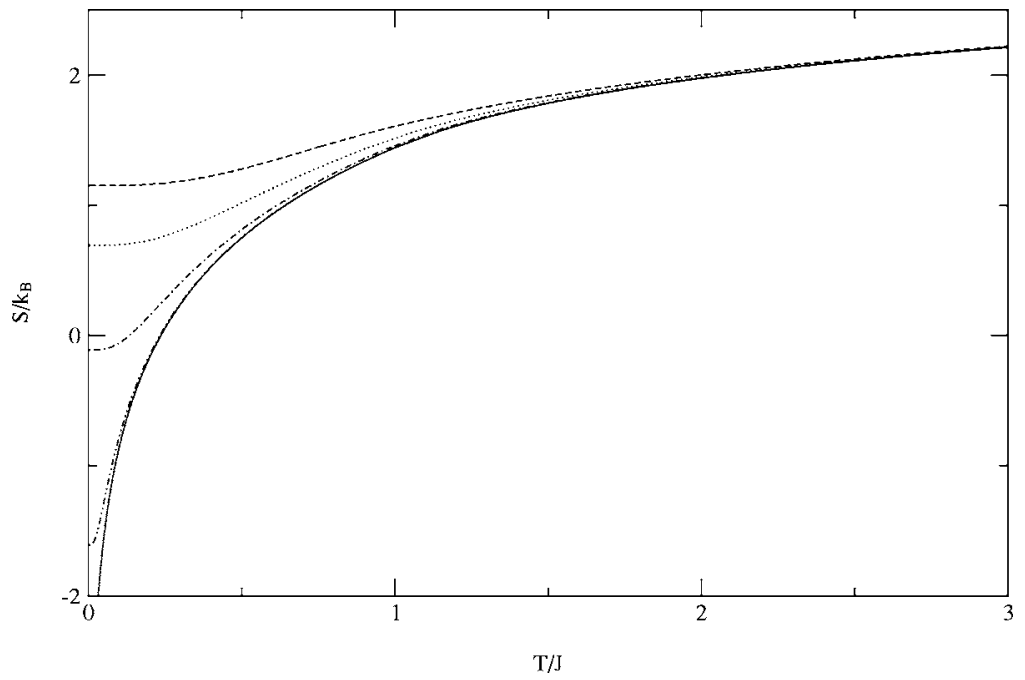

FIG. 4. Entropy as a function of temperature for different values of the moment of inertia $I$ $=J$ (dash), $I=5 J$ (dash-double-dotted line), $I$ $=10 J$ (dash-dotted line), $I=\infty$ (solid line).

$$
\begin{aligned}
\beta F_{\text {class }}= & \frac{1}{2} \ln \left[\beta \mu+\sqrt{(\beta \mu)^{2}-(\beta J)^{2}}\right] \\
& +\frac{1}{4} \frac{\beta \mu-\sqrt{(\beta \mu)^{2}-(\beta J)^{2}}}{\beta \mu+\sqrt{(\beta \mu)^{2}-(\beta J)^{2}}}-\mu \beta-\ln \frac{\sqrt{\beta J}}{2} .
\end{aligned}
$$

The expression in Eq. (35) coincides with the results for the classical spherical spin glass in Refs. 1 and 4, but the free energy in Eq. (36) differs due to the last logarithmic contribution. This originates in the choice of the normalization constant $\mathcal{N}$. If we had chosen $\mathcal{N}$ following Feynman's prescription, ${ }^{16,17}$ we would have obtained unphysical results in the quantum regime for finite values of $I$, like a negative specific heat at low temperatures. ${ }^{19}$ The chemical potential $\mu$ decreases with temperature until it reaches the value $\mu_{c}=J$ (Ref. 20) at the critical temperature $T_{c}$; for lower temperatures the spherical condition no longer holds and $\mu$ sticks to this value. We show in Fig. 1 the solution for $\mu$ as a function of $T$ for different values of $I$. The critical temperature is obtained by setting $\mu=\mu_{c}=J$ in Eq. (32). We show in Fig. 2 the phase diagram for $T_{c}$ as a function of $I . T_{c}$ decreases with
$1 / I$ and vanishes at the critical value $I_{c}=\frac{16}{9 \pi^{2} J}$. This result coincides with previous references. ${ }^{9,10}$ Close to this value we find $T_{c}=\sqrt{\frac{J\left(I-I_{c}\right)}{3 I_{c}^{2}}}$. We show in Figs. 3-5 results for the free energy, entropy, and specific heat as functions of temperature for different values of $I$. We observe that the entropy remains finite when $T \rightarrow 0$ for any finite value of $I$, while we recover the usual divergence $S \rightarrow-\infty$ in the classical spherical spin glass $\operatorname{model}^{1,4}$ when $I \rightarrow \infty$. The specific heat vanishes at $T$ $=0$ for finite values of $I$, as expected in quantum systems. ${ }^{12-15}$ The dynamic susceptibility is the response to the field $H\left(\omega_{n}\right)$ and we obtain from Eq. (27)

$$
\chi\left(\omega_{n}\right)=\frac{\omega_{n}^{2} I+2 \mu-\sqrt{\left(\omega_{n}^{2} I+2 \mu\right)^{2}-4 J^{2}}}{2 J^{2}} .
$$

The static susceptibility

$$
\chi(0)=\frac{\mu-\sqrt{\mu^{2}-J^{2}}}{J^{2}}
$$

satisfies the Curie law for high temperatures and exhibits the usual cusp at the critical temperature. It is shown in Fig. 6 for different values of $I$. The dynamic susceptibility at $T=0$

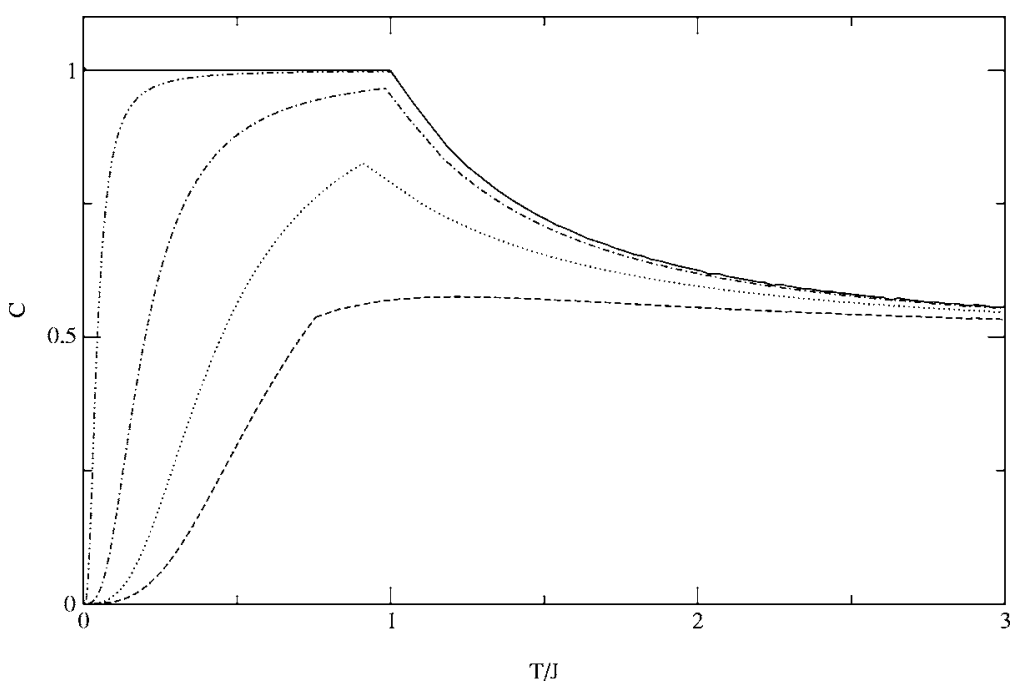

FIG. 5. Specific heat as a function of temperature for different values of the moment of inertia $I=0.4 J$ (dashed line), $I=J$ (dash-dotted line), $I$ $=10 \mathrm{~J}$ (dash-double-dotted line $), I=\infty$ (solid line $)$. 


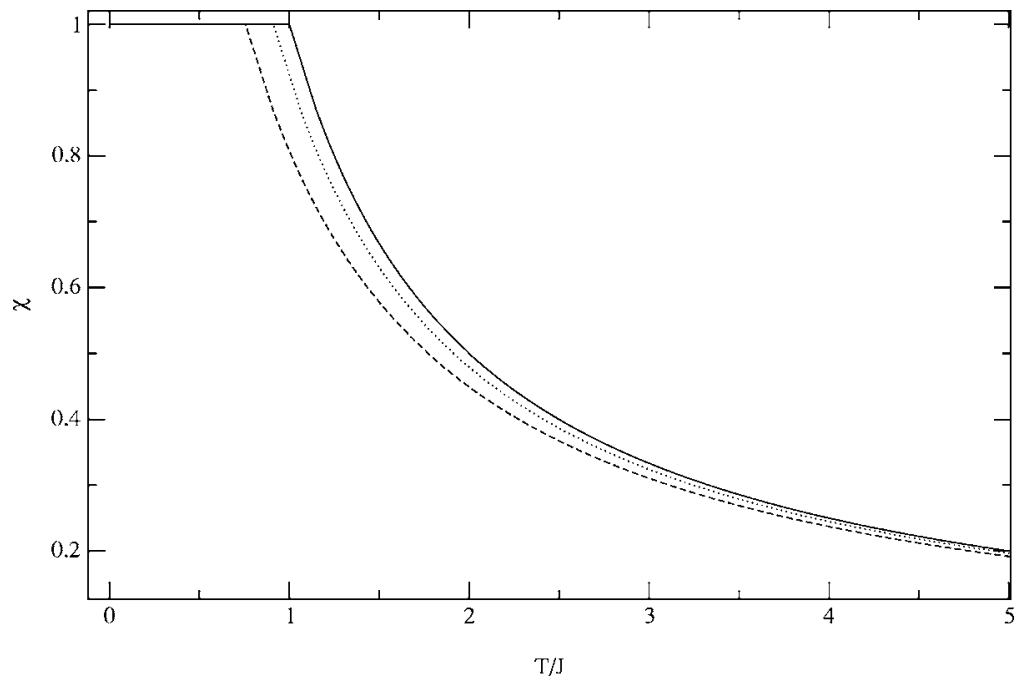

FIG. 6. Static susceptibility as a function of temperature for $I=0.4 J$ (dashed line), $I=J$ (dashdotted line), $I=\infty$ (solid line).

is obtained by setting $i \omega_{n} \rightarrow \omega+i \delta$ in Eq. (37). For $\Delta=\mu-J$ $\geqslant 0$ and $I=I_{c}$ we obtain that $\operatorname{Im} \chi(\omega)$ satisfies the scaling relation

$$
\operatorname{Im} \chi(\omega)=\operatorname{sgn} \omega|\omega|^{\mu^{\prime}}\left(\sqrt{I_{c}} / J\right) \Phi\left(\omega \sqrt{\frac{I_{c}}{J^{2} \Delta}}\right),
$$

with the exponent $\mu^{\prime}=1$ and the scaling function $\Phi(x)$ $=\sqrt{1-x^{-2}}$, which agrees with the results of Ref. 10 .

\section{CONCLUSIONS}

The quantum spherical spin glass was studied within the annealed average, which is exact due to the internal BRST supersymmetry of the model as was discussed in Sec. I. Our results for the phase diagram coincide with those obtained by Ye et al. ${ }^{10}$ with the replica method and by Shukla and Singh ${ }^{9}$ using the semicircular law. However, a substancial difference exists between our results for the free energy, entropy, and specific heat and those presented by Shukla and Singh ${ }^{9}$ The results obtained with quantum functional integrals methods are very sensitive to the use of the correct normalization and measure, ${ }^{16,17}$ and we set the normalization constant in our expression for the free energy so as to recover a finite limit when $I \rightarrow \infty$. As a result we obtain a finite entropy at low temperatures and a vanishing specific heat for finite values of the rotors moment of inertia $I$, as is expected in quantum spherical models. ${ }^{12-15}$ In the limit $I \rightarrow \infty$ we recover the known negative, infinite entropy and a constant specific heat at $T=0$. Also at $T=0$ we derive the scaling behavior for the dynamical susceptibility that agrees with Ye et al. ${ }^{10}$

\section{APPENDIX}

We refer here to Refs. 2 and 3 to give a concise and brief description of Grassmann variables and superalgebra. We indicate complex Grassmann variables by Greek letters, and these anticommuting variables satisfy

$$
[\chi, \gamma]_{+}=0, \quad \chi^{2}=\chi^{* 2}=0,
$$

$$
\begin{gathered}
\chi^{* *}=-\chi, \\
(\chi \gamma)^{*}=\chi^{*} \gamma^{*}, \\
\left(\chi^{*} \chi\right)^{*}=\chi^{* *} \chi^{*}=-\chi \chi^{*}=\chi^{*} \chi
\end{gathered}
$$

and the integrals

$$
\begin{gathered}
\int d \chi^{*} \chi^{*}=\int d \chi \chi=1, \\
\int d \chi=\int d \chi^{*}=0,
\end{gathered}
$$

which leads to

$$
\int \prod_{i} d \chi_{i}^{*} d \chi_{i} \exp \sum_{i j} \chi_{i}^{*} M_{i j} \chi_{j}=\left\|M_{i j}\right\| .
$$

A four-component supervector $\underline{\varphi}$ and a $4 \times 4$ supermatrix $\mathbf{M}$ have commuting and Grassmann components

$$
\begin{gathered}
\underline{\varphi}=\left(\begin{array}{c}
R_{1} \\
R_{2} \\
\chi / \sqrt{2} \\
\chi^{*} / \sqrt{2}
\end{array}\right), \\
\mathbf{M}=\left(\begin{array}{ll}
\underline{a} & \underline{\sigma} \\
\underline{\rho} & \underline{b}
\end{array}\right),
\end{gathered}
$$

where $\underline{a}$ and $\underline{b}$ are $2 \times 2$ matrices of commuting elements while $\underline{\sigma}$ and $\underline{\rho}$ are $2 \times 2$ matrices of Grassmann elements. Taking into account the change of sign involved in complex conjugation from Eq. (A1) the superadjoint must be defined:

$$
\begin{gathered}
S \underline{\varphi}^{\dagger}=\left(R_{1} R_{2} \chi^{*} / \sqrt{2}-\chi / \sqrt{2}\right), \\
S \mathbf{M}^{\dagger}=\left(\begin{array}{cc}
\underline{a}^{\dagger} & \underline{\rho}^{\dagger} \\
-\underline{\sigma}^{\dagger} & \underline{b}^{\dagger}
\end{array}\right) .
\end{gathered}
$$


${ }^{1}$ Alba Theumann, J. Phys. A 20, 25 (1987).

${ }^{2}$ K. Efetov, Supersymmetry in Disorder and Chaos (Cambridge University Press, Cambridge, England, 1997).

${ }^{3}$ P. Ramond, Field Theory: A Modern Primer (Benjamin/ Cummings, New York, 1981).

${ }^{4}$ J. M. Kosterlitz, D. J. Thouless, and R. C. Jones, Phys. Rev. Lett. 36, 1217 (1976).

${ }^{5}$ Alessia Annibale, Giulia Gualdi, and Andrea Cavagna, J. Phys. A 37, 11311 (2004).

${ }^{6}$ Andrea Cavagna, Juan P. Garrahan, and Irene Giardina, J. Phys. A 32, 711 (1999).

${ }^{7}$ A. Crisanti, L. Leuzzi, G. Parisi, and T. Rizzo, Phys. Rev. B 68, 174401 (2003).

${ }^{8}$ C. Becchi, A. Rouet, and R. Stora, Ann. Phys. (N.Y.) 98, 287 (1976).

${ }^{9}$ Prabodh Shukla and Surjit Singh, Phys. Lett. 81A, 477 (1981).

${ }^{10}$ J. Ye, S. Sachdev, and N. Read, Phys. Rev. Lett. 70, 4011 (1993).

${ }^{11}$ Subir Sachdev, Quantum Phase Transitions (Cambridge University Press, Cambridge, England, 2001).

${ }^{12}$ Th. M. Nieuwenhuizen, Phys. Rev. Lett. 74, 4289 (1995); 74,
4293 (1995); Th. M. Nieuwenhuizen and Felix Ritort, Physica A 250, 8 (1998).

${ }^{13}$ Thomas Vojta, Phys. Rev. B 53, 710 (1996).

${ }^{14}$ Yuhai Tu and Peter B. Weichman, Phys. Rev. Lett. 73, 6 (1994).

${ }^{15}$ M. H. Oliveira, M. D. Coutinho-Filho, and E. P. Raposo, Phys. Rev. B 72, 214420 (2005).

${ }^{16}$ (a) John W. Negele and Henry Orland, Quantum Many Particles (Addison-Wesley, Reading, MA, 1987); (b) V. N. Popov, Functional Integrals and Collective Excitations (Cambridge University Press, Cambridge, England, 1987).

${ }^{17}$ R. P. Feynman and A. R. Hibbs, Quantum Mechanics and Path Integrals (McGraw-Hill, New York, 1965).

${ }^{18}$ Daniel J. Amit, Field Theory, the Renormalization Group and Critical Phenomena (World Scientific Publishing, Singapore, 1984).

${ }^{19}$ Pedro Castro Menezes Xavier de Melo e Silva, Master thesis, Instituto de Fisica, Universidade Federal do Rio Grande do Sul, Brazil, 2005.

${ }^{20}$ T. H. Berlin and M. Kac, Phys. Rev. 86, 821 (1952). 